Erler, Alexandre and Müller, Vincent C. (forthcoming 2021), 'The ethics of biomedical military research: Therapy, prevention, enhancement, and risk', in Daniel Messelken and David Winkler (eds.), Health care in contexts of risk, uncertainty, and hybridity (Berlin: Springer), 1-20.

[August 2021, http://www.sophia.de]

\title{
The Ethics of Biomedical Military Research: Therapy, Prevention, Enhancement, and Risk
}

\author{
By Alexandre Erler and Vincent C. Müller
}

\begin{abstract}
What proper role should considerations of risk, particularly to research subjects, play when it comes to conducting research on human enhancement in the military context? We introduce the currently visible military enhancement techniques (1) and the standard discussion of risk for these (2), in particular what we refer to as the 'Assumption', which states that the demands for risk avoidance are higher for enhancement than for therapy. We challenge the Assumption through the introduction of three categories of enhancements (3): therapeutic, preventive, and pure enhancements. This demands a revision of the Assumption (4), alongside which we propose some further general principles bearing on how to balance risks and benefits in the context of military enhancement research. We identify a particular type of therapeutic enhancements as providing a more responsible path to human trials of the relevant interventions than pure enhancement applications. Finally, we discuss some possible objections to our line of thought (5). While acknowledging their potential insights, we ultimately find them to be unpersuasive, at least provided that our proposal is understood as fully non-coercive towards the candidates for such therapeutic enhancement trials.
\end{abstract}

\section{Introduction: Human Enhancement in the Military}

Militaries around the world share an interest in making soldiers more effective at performing their tasks. One path towards that goal is to use modern technology and medical science to improve soldiers' physical and mental capacities - what has been termed "human enhancement". Military enhancements, as we will understand them in this chapter, can be defined as interventions, typically involving some form of biomedical technology, that either:

1. Improve aspects of a soldier's functioning beyond what is considered "normal"; or

2. Give a soldier new capabilities that "normal", non-enhanced humans do not possess.

Enhancements are often contrasted with therapeutic interventions: this is known as the therapyenhancement distinction. While the usefulness of that distinction has been challenged by some authors (e.g. Savulescu et al., 2011), we adopt it here as a conceptual tool we consider helpful, although we will challenge the common assumption that it posits a strict dichotomy between 
the two different types of intervention. ${ }^{1}$

We understand therapeutic interventions to also improve certain aspects of a person's functioning, yet - unlike pure enhancements - in a way that either restores or maintains health or normal functioning. To limit the scope of our discussion, we will also assume that both therapies and enhancements are integrated into a person's body and bodily functioning to a degree that devices regarded as mere "tools" are not, even when their effects are comparable to the former kinds of intervention (on this, we follow Lin, Mehlman and Abney, 2013). For example, a powered exoskeleton that increases a soldier's ability to handle heavy loads does not seem to truly become part of a soldier's body, but rather constitutes an addition to it that amplifies the impact of the actions it takes - it is thus not an "enhancement" according to the definition we use here. A prosthetic limb, by contrast, is required for the integrity of an amputee's body, and can therefore meet the integration requirement, even though it remains a non-invasive intervention. And while brain stimulation devices are typically not so integrated (except when they are implanted into the skull), the proximate cause of their effects on mental function - alterations in the properties and behaviors of neurons - certainly is. ${ }^{2}$

In our discussion, we will mostly be referring to the US military for illustrative purposes, given the wealth of evidence it provides about both enhancement use and cutting-edge research (conducted mainly through the Defense Advance Research Projects Agency or DARPA). Among military enhancements in the first of the two categories distinguished above, some are meant to improve a soldier's physical functioning: capacities like endurance or strength. Steroids like testosterone have been shown to increase muscle strength in healthy users, and there is evidence that their covert use among US soldiers is relatively widespread, although their safety profile is controversial, and their use is currently illegal in the US military without a prescription (Peltier and Pettijohn, 2018). On a more futuristic note, some have speculated that regenerative medicine might also have the potential to enhance soldiers' physical performance, for instance via the creation of artificial organs using technologies like 3D bioprinting, the original purpose of which would be therapeutic, yet which might eventually

\footnotetext{
${ }^{1}$ We may note that those who do not define enhancements in contrast to therapy do not posit such a dichotomy. If, for instance, enhancements are understood as biomedical interventions that increase a person's chance of living a good life in a given set of circumstances, most therapies will count as a subset of enhancements.

${ }^{2}$ Even though we take this point about integration to be practically useful for the purpose of our discussion, we are not strongly committed to it, and it is not crucial that our readers' intuitions should be fully in line with it.
} 
surpass "natural" human organs after further refinements (Campobasso, 2015). Finally, speculative applications of recent advances in genome editing technology, and particularly CRISPR/Cas9, to enhance physical capacities on the battlefield have also been described: they include facilitating muscle gain, and improving the ability to see in low light conditions (Greene and Master, 2018).

Other military enhancements are focused on improving mental function: capacities like wakefulness, attention, memory, or processing speed. The use of wakefulness-promoting substances (initially developed for therapeutic purposes) already has a long history in the US military (and others), with drugs like amphetamines and, more recently, modafinil (Mehlman, 2015). For a number of years already, DARPA has been running various programs aimed at discovering new ways of enhancing soldiers' mental capacities. A recent example is the Next Generation Nonsurgical Neurotechnology (N3) program, which seeks to develop either noninvasive or "minutely invasive" forms of brain-computer interface (BCI) that would endow healthy soldiers with new abilities, such as controlling active cyber defense systems, and swarms of unmanned aerial vehicles, using only their thoughts (DARPA, 2019).

DARPA-funded research also includes more invasive interventions into the brain, such as neural prostheses and implants: on example is the Restoring Active Memory (RAM) program, which seeks to develops tools to restore normal memory in military personnel suffering from brain injury or illness (DARPA, 2018). Nonetheless, as illustrated by the N3 program, the agency is seeking to limit the scope of application of such invasive devices to purely therapeutic ones, and ultimately to make reliance on invasive interventions altogether redundant - a perfectly sensible policy of risk minimization. Still, it remains an open question at this stage whether non-invasive interventions will be able to match invasive ones (which likely allow for higher resolution readings) in terms of the outcomes they make possible.

A final relevant distinction among military enhancements is between reversible and irreversible (or less easily reversed) ones - a distinction that largely overlaps with that between invasive and non-invasive interventions. The enhancement effects of substances like caffeine, modafinil, or transcranial direct current stimulation or tDCS, can all be reversed quite easily, within a reasonably short time frame, by ceasing to use the intervention. (Assuming they have no long-term effects.) By contrast, removing an artificial "super organ", or a neural implant, is a much more delicate procedure, making such invasive interventions less easily reversible 
(although not completely irreversible, unlike a more extreme case such as neurosurgery). We may further note that the reversible/irreversible contrast applies not only to the enhancement effects of the interventions we are discussing, but also to any unintended side effects they might have. It seems plausible to assume that there will be some correlation between the extent to which a particular intervention is reversible, and the extent to which its side effects are. Nonetheless, this correlation need not be perfect. It is thus possible for a reversible intervention, such as a certain psychoactive drug, to have irreversible unintended side effects, for instance if it were to induce a stroke in the user.

\section{The issue of risk in enhancement research}

One key consideration in the context of military enhancement research relates to the risks that such research presents for participants in the relevant clinical trials, and how they compare to the benefits that they might gain from the research - what is known as the intervention's riskbenefit ratio (RBR) for the participants concerned. The basic idea is that the intervention being tested must offer benefits of sufficiently high magnitude to justify the risks it will impose on the research subjects. (To clarify, we understand "military enhancement research" to be aimed at developing interventions that will help members of the armed forces in the completion of their duties. Yet such research can in principle involve civilians as research subjects, especially in its initial stages, as illustrated by some of the studies funded by DARPA.)

When it comes to enhancement research, a relatively common belief, which we shall simply refer to as the Assumption, states the following:

The Assumption: Because those who are ill or disabled start from a lower baseline of health and have less to lose than healthy individuals seeking to become "better than well", therapeutic interventions will typically have a more favorable RBR than enhancements. In light of this, responsible practices will entail a lower tolerance for potential bad outcomes, or alternatively, the potential for greater benefits to research subjects, in the case of enhancement research (see e.g. Agar, 2004; McGee and Maguire, 2007).

Maxwell Mehlman and Jessica Berg have challenged the Assumption, arguing that "some enhancement benefits may be perceived as more valuable than medical benefits". As an example, they contrast an intervention that substantially boosted normal cognitive capacity 
with "a substance to treat a minor skin irritation" (Mehlman and Berg, 2008, p. 550). Their point is correct, and does call into question the first part of the Assumption: at the very least, it shows that its first claim cannot simply be derived from the very nature of therapies and enhancements, contrary to what its proponents are assuming. The fact that the benefits conferred by enhancements can in principle surpass those of some therapies shows that, even if research on the former category of interventions typically carries greater risks for subjects than therapeutic research, we still cannot infer that its RBR (risk-benefit ratio) must typically be worse, since that RBR is a function of the magnitude of both risks and benefits involved. That said, Mehlman and Berg's objection does not seem to contradict the second part of the Assumption, since it seems compatible with the claim that enhancement research must involve less serious potential bad outcomes, assuming comparable benefits, than therapeutic research, or greater potential benefits for comparably bad potential outcomes.

To take Mehlman and Berg's critique into account, we might thus try to reformulate the Assumption in the following manner:

The Revised Assumption, version 1: Because [...] "better than well", therapeutic interventions will typically have a more favorable RBR than enhancements when they offer benefits of comparable magnitude. In light of this, etc.

However, this first revised version of the Assumption can also be challenged. First, even if one were to accept its validity in most ordinary contexts, one might nevertheless question whether it also holds in the military one. Indeed, soldiers involved in combat missions face great risks, which enhancements might conceivably help mitigate (as suggested by Mehlman and Li, 2014). Furthermore, it seems possible that, at least sometimes, enhancements will allow to reduce such risks to a greater degree than would be the case if a soldier were to use a similar intervention for therapeutic purposes. To take a futuristic example, an artificial lung that simply restored an injured soldier back to "normal" functioning might not reduce combat-related risk to the same degree as a "super" lung that enhanced endurance in a healthy subject, making him or her less likely to be captured or shot. The RBR of the enhancement intervention thus need not be worse than that of the therapeutic transplant, even assuming they would yield benefits of comparable magnitude, because the greater potential loss faced by a healthy research subject might be compensated for by the greater risk-reducing impact of the enhancement. In the context of armed conflict, one might suppose that this may be true of a number of other 
enhancements. Of course, this argument will only hold water if the enhancement in question is tested within that particular context, and will no longer apply if the research is instead conducted in a much less dangerous environment (say, military exercises).

A second, stronger challenge to the first revised version of the Assumption, one that extends beyond the military context, contends that it is built on a false dichotomy between therapy and enhancement. The next section spells out this line of argument further, by distinguishing between three different categories of enhancements.

\section{A tripartite distinction between enhancements}

The therapy-enhancement distinction is often understood as contrasting two fundamentally different, and mutually exclusive categories of interventions. Even though it is accepted that there might not be a sharp dividing line between the two, so that some interventions might not confidently be fit into one category rather than the other, the assumption tends to be that an intervention qualifies either a therapy or enhancement (or neither), but never as both. However, as some authors have noticed, this assumption seems questionable - even if the distinction is cashed out in the (common) way we have outlined above. A more accurate picture involves recognizing that while some enhancements may indeed fit the standard paradigm by being completely divorced from therapy, others do not. Enhancements of the latter kind also have therapeutic or preventive effects. We therefore propose to distinguish between the following three categories of enhancements:

a. "Therapeutic": Therapeutic enhancements either bring someone's initially subnormal functioning beyond the threshold of "normality" (or health), or restore normality by conferring new abilities (e.g. Wolbring \& al., 2013). Examples of the former kind include a short-sighted person who undergoes laser eye surgery to correct that impairment, and ends up with better than 20/20 vision (a description that already applies to actual people); or a pair of prosthetic legs that allowed an amputee to run faster than they could with their original, "normal" legs - a phenomenon that is, if not a current reality (as was once assumed about former sprinter Oscar Pistorius), at least a plausible near-term development. A contemporary example of the latter kind is provided by brain implants that allow paralyzed patients to control a computer using only their thoughts 
(e.g. Nuyujukian et al., 2018). Such an intervention can be described as restoring one type of normal ability (control of a computer) by conferring a new, "superhuman" one (direct control of the device via thought).

Future technological developments can be expected to yield new forms of therapeutic enhancement that could be relevant to the military. We have mentioned the example of artificial organs: once they get sufficiently perfected to be suitable for transplantation, they will hopefully match "natural" donated organs in terms of their benefits for patients, before ultimately surpassing them after further refinements. The ultimate achievement in this endeavor would be "super" organs conferring a higher level of functioning than a person's natural organs could ever have attained, even in the absence of pathology. Implanting such an organ into a patient to remedy their organ failure will result in a therapeutic enhancement. An analogous state of affairs might also come to be with regards to brain functioning: for example, a patient with memory deficits as a result of damage to the hippocampus could conceivably, if fitted with a cutting-edge memory prosthesis, achieve a better level of recall than she might ever have enjoyed with her "normal" hippocampus.

b. "Preventive": Preventive enhancements forestall the advent of disease or disability by improving upon already normal functioning (e.g. Brock, 1998; Erler, 2017). A paradigmatic example of this category of enhancements would be vaccines, which enhance normal immunity to prevent disease - especially those that are not yet routinely used in the population, and therefore cannot plausibly be said to have redefined what counts as "normal functioning". The military context provides good illustrations of such interventions, such as the anthrax vaccine administered by the US military to its personnel as part of the Anthrax Vaccine Immunization Program from 1998 to 2008. Future developments in biomedical science might open up new ways of enhancing soldiers to protect them from normal vulnerability to injury and illness. Greene and Master (2018) thus anticipate that genome editing via CRISPR/Cas9 might be used to confer immunity to new biological agents.

We have previously mentioned the risk mitigation potential of enhancements for soldiers involved in dangerous combat missions. This raises the question whether all enhancements that somehow reduce a soldier's risk of dying or getting injured should 
be considered preventive enhancements. Although this is to a large degree a linguistic point, we suggest that a negative answer is more plausible: on our understanding of such enhancements, their protective effects must be direct, not indirect. The protection against disease afforded by a vaccine does meet that requirement. By contrast, the reduction in risk that might result from enhanced cognitive capacities, which might for instance improve strategic planning, does not. In such a scenario, the proximate cause of the non-occurrence of death or injury would be, for example, a certain kind of evasive action, and not the superior cognitive capacities that had made such action possible. We do agree, however, that in individual instances where we can reasonably predict that a given intervention will have indirect preventive effects, these should be included into the calculation of that intervention's RBR for the subject concerned.

c. "Pure": Pure enhancements, which might fit the paradigm that many have in mind when they hear the term "enhancement", improve upon already normal functioning to a level that is either on the upper end of the natural distribution, or goes beyond it entirely, and do not either remedy or prevent any pathological condition. A person with 20/20 vision who underwent laser eye surgery to improve it further would provide an illustration of such an enhancement, as would someone who, thanks to a drug or brain implant, managed to boost their normal memory. As we have seen, such enhancements could conceivably - and perhaps often - reduce the risk of harm faced by the soldier, albeit indirectly. ${ }^{3}$

We will now see that this tripartite distinction has implications for the validity of the Assumption, and the ethics of military enhancement research.

\section{Re-thinking risks and benefits in the context of enhancement research}

The tripartite distinction we introduced in the previous section highlights a key limitation of

\footnotetext{
${ }^{3}$ On the other hand, it is also conceivable that, depending on the circumstances, having received a therapeutic or a pure enhancement that increased one's effectiveness in combat might actually end up increasing the soldier's risk of harm, at least in one respect. Suppose for example that the enemy were able to identify soldiers who had received such enhancements: they might then view them as presenting a special threat, to be dealt with as a matter of priority. Since it is not clear that this is a particularly likely possibility, however, we leave it aside for the sake of our discussion.
} 
the Assumption, even in its first revised version: namely, it illegitimately presupposes that any potential beneficiary of an enhancement will be "a healthy individual seeking to become better than well", that is, that all enhancements are pure enhancements. As we have just seen, this is not the case. We therefore propose the following, second reformulation of the Assumption, so as to avoid positing a false dichotomy between therapies and enhancements, while also incorporating the above remarks about the potential risk-reducing effects of military enhancements in combat situations:

The Revised Assumption, version 2: Because [...] "better than well", interventions with therapeutic or preventive benefits will typically have a more favorable RBR than pure enhancements, when they offer benefits of comparable magnitude. In light of this, responsible practices will entail a lower tolerance for potential bad outcomes, or alternatively, the potential for greater benefits to research subjects, in the case of pure enhancement research (at least in cases where such research is conducted in reasonably safe environments).

The counterpart of this is that pure therapies will also typically have a better RBR than therapeutic (but not necessarily preventive) enhancements, when they offer benefits of comparable magnitude. That is the second grain of truth in the original Assumption. Indeed, if two therapies lead to comparable gains in functioning for research subjects, and only one of them is a therapeutic enhancement, it follows that the recipient of the latter must have started at a higher level of health or functioning than the recipient of the pure therapy, and therefore had more to lose. That said, for the reasons outlined by Mehlman and Berg, it does not follow that pure therapies will, as a general rule, have a more favorable RBR than any of the three categories of enhancement we have distinguished. Perhaps that is in fact the case with regards to one or more of these categories; nonetheless, if it is so, it will still not follow from the very nature of enhancements as contrasted with pure therapies, and would need to be established by engaging in a comprehensive empirical survey of the relevant interventions (which would allow us to compare the RBRs of existing interventions, but of course not of all possible ones).

For similar reasons, one cannot confidently claim that therapeutic enhancements will typically show a better RBR than pure enhancements. While the latter will typically be riskier than the former, they might also, at least sometimes, offer greater benefits. However, it does seem that certain kinds of therapeutic enhancements will indeed show a RBR superior to that of pure 
enhancements. And those of particular relevance in the context of military enhancement research will be those displaying a superior RBR while producing outcomes comparable to those of pure enhancements. For instance, if cutting-edge prosthetic limbs ever surpass normal human limbs in their functionality, the beneficiaries of such devices who became amputees as a result of an injury or disease will achieve the same level of performance as hypothetical healthy subjects looking for an extra physical edge via elective amputation, but they will enjoy a better RBR, since they started from a disabled state. Similar remarks will apply to the tetraplegic recipient of an invasive BCI, vs. a healthy recipient. Both might gain the same kind of new abilities that organizations like DARPA are interested in developing, yet the former might enjoy a better RBR.

The common denominator of the therapeutic enhancements in question seems to be, first, that they are all invasive in nature. The preceding remarks might in principle apply to non-invasive interventions, too, such as non-invasive BCIs. However, this will only be so if the modus operandi of the device (say, electrical fields) does present some degree of risk to research subjects. If it involves little to no risk, then uses of such devices for therapeutic enhancement will no longer enjoy a more favorable RBR. As we mentioned in section 1, non-invasive interventions will in any case tend to be less risky (partly because more easily reversible) than invasive ones, so that we will have a strong reason to prefer the former when they offer similar benefits to the latter. However, in at least some cases, we can expect invasive interventions to have an edge in terms of their effectiveness.

A second commonality is that when such interventions are applied to a subject in a pathological condition, that condition does not hamper in any way the enhancing effect of the intervention, compared to what would happen if it were applied to a healthy subject. One reason why it might be so is that the intervention involves the full replacement of a certain body part with a new, "enhancing" one, so that it ultimately does not make a difference whether the original part was dysfunctional (or missing) or not. Another possible reason is that the intervention is endowing the recipient with entirely new abilities, such as control of drones and other military vehicles by thought alone. While implanting such a device into the brain of a paralyzed subject might also help compensate for some of the loss in her motor function, she would still enjoy these new abilities to the same extent as a fully healthy recipient would.

When it comes to (usually invasive) interventions that fit the description just given, we can 
therefore conclude that, when we have the choice between testing a new intervention of this kind as a therapeutic or pure enhancement, we should, from the perspective of securing the best possible RBR for research subjects, choose the former. This goes one step beyond the well-established idea that, whenever possible, we should test invasive interventions like neural implants on patients with pathological conditions first, for purely therapeutic purposes, before considering - assuming it is ethical to do so - enhancement uses involving healthy subjects (which could benefit from the knowledge, e.g. about the intervention's safety profile, acquired from the therapeutic research). Of course, the goal of securing the best RBR we can achieve is only one of several relevant considerations in research ethics (and it thus need not automatically translate into an all-things-considered judgment). Our discussion thus leaves aside a number of other issues, such as what constitutes truly informed consent from subjects in this context, or broader questions about the ethics of human enhancement, such as the possible value of the "natural". Nonetheless, the said goal arguably remains a very important consideration here.

Ideally, such interventions would help soldiers return to active duty after a period of physical training, giving them an extra edge in the performance of their tasks. In cases where a soldier cannot return to active duty, it is still conceivable that this person might be given the opportunity to enroll in a trial for a therapeutic enhancement. However, assuming the intervention in question were not primarily designed for use in military operations, it would no longer count as military enhancement research - even though some might argue that ill or disabled veterans should get easier access to such trials as a reward for serving their country. We return to this potentially contentious issue in subsection 5.3.

Having outline our proposed revisions to the Assumption, as well as our core line of argument regarding the relevance of certain therapeutic enhancements for the sake of optimizing the RBR of military enhancement research, we now turn to some potential objections to it.

\section{Possible objections}

\subsection{Therapeutic enhancements need not all have a more advantageous $R B R$}

To begin with, it is clear that the tendency to show a better RBR when used as therapeutic rather than pure enhancements will not apply to all potential military enhancements. For 
example, a number of interventions will, if equally strong in intensity, allow for a lower level of performance if used on an ill or disabled subject, rather than on a healthy one. In such cases, using the intervention as a therapeutic enhancement will require resorting to greater intensity to reach results comparable to its use as a pure enhancement. A person with excessive sleepiness who needed a daily dose of $200 \mathrm{mg}$ of modafinil to reach a certain enhancement level (say, in terms of wakefulness or executive function) might thus not enjoy a better RBR than a person with no such condition who only needed $100 \mathrm{mg}$ daily to reach the same enhancement level.

Somewhat similarly, a subject implanted with a neuroprosthesis for memory deficits resulting from brain injury, who needed strong electrical stimulation to reach a certain level of enhanced memory, may or may not enjoy a better RBR than a subject with normal memory who only needed less intense stimulation from the same prosthesis to reach the same ultimate level of recall. On the one hand, the risks associated with surgery would likely be the same in both cases, which would boost the therapeutic enhancement's RBR compared to that of the pure enhancement. Yet on the other hand, this advantage might be cancelled out if the stronger stimulation required for the therapeutic enhancement presented greater risks than the weaker stimulation associated with the pure enhancement. If so, the commitment to optimize the balance between risks and benefits for research subjects will not support preferring therapeutic enhancement applications, rather than pure enhancement ones, when conducting human trials of such interventions.

What is more, some of the conditions that therapeutic enhancements might in principle target are currently disqualifying for recruitment in the military. For example, a diagnosis of Attention Deficit Hyperactivity Disorder that required the use of prescription medication in the previous 24 months, and a history of narcolepsy are treated as disqualifying conditions by the US Army (Office of the Under Secretary of Defense for Personnel and Readiness, 2018). Similarly, a candidate for genome editing who suffered from muscular dystrophy would also be ineligible for military enrollment. Even if it were in principle possible for this person to end up with an enhanced ability for muscle gain as a result of the intervention, by having an "optimal" rather than just "normal" genetic variant (from the perspective of muscle gain) inserted to replace her initial, pathological one, this possibility would be irrelevant in actual practice - at least in the current state of affairs. 
These remarks do not refute the claims we presented in the previous section, but simply emphasize that their scope of application is limited to a certain type of interventions, those fitting the description we have outline above.

\subsection{Coercing/targeting vulnerable subjects}

The suggestion that ill or disabled subjects should, all else being equal, be preferred for trials of (mostly invasive) new interventions with prospective military enhancement applications, might strike some as perverse. They might object that it would mean unfairly targeting people in a vulnerable position, who, given their medical needs, will face stronger psychological pressures to take part in such trials than healthy subjects with no such needs. The existence of such pressures might especially be a concern when prospective subjects are members of the armed forces, who are expected to obey orders from their superiors. ${ }^{4}$ Alongside this worry about potential coercion, there might be the sense that researchers should not unnecessarily impose extra risks on people who already have significant medical needs (and are, in that respect, worse off than others), and that our proposal precisely entails doing so.

We agree that such concerns are legitimate. However, we also think that they mostly apply to cases where a subject is forced to participate in the relevant trial, and faces sanctions for refusing. We certainly do not mean to suggest that anyone, whether civilians or members of the armed forces, should ever be subjected to such pressures. ${ }^{5}$ On the contrary, participants in such trials should all enjoy the standard protections accorded to research subjects, guaranteeing that their participation is fully voluntary. Those who do not wish to enroll in the trial should instead be given access to the most solidly established, purely therapeutic intervention for their condition. Furthermore, while a policy focused on promoting the best possible RBR for research subjects does strike us as sensible overall, it should nonetheless remain constrained by the recognition of an absolute threshold of risk (to be determined by experts) that no ethical research should cross, regardless of the benefits it might hold. It is therefore conceivable that

\footnotetext{
4 The use of the term "vulnerable" is, of course, not meant to deny in any way the resilience that members of the military so often display in the face of injury and other kinds of adversity.

${ }^{5}$ As Lin and colleagues mention, it is possible to argue that there are exceptions to this rule: one illustration would be the administration of somewhat experimental vaccines to American soldiers during the first Gulf War (Lin, Mehlman and Abney, 2013; although these authors also question whether this practice should be regarded as a formal military experiment). However, it seems that such exceptions will typically involve preventive rather than therapeutic enhancements.
} 
trials of certain exceptionally risky therapeutic enhancements might be impermissible, even if the RBR of those interventions were superior to that of purely therapeutic alternatives for the same condition.

A related objection might be that even offering a therapeutic enhancement, as an optional alternative to a pure therapy, to a subject with medical needs would violate the principle of "clinical equipoise" (Weijer and Miller, 2004), which does not allow researchers to test an experimental intervention that they confidently expect not to provide any extra therapeutic benefits compared with the alternative that medical experts would agree is appropriate in the circumstances, while potentially imposing greater risks on research subjects. In response, we agree that researchers should be reasonably confident that any intervention they might test as a therapeutic enhancement will answer the patient's medical needs about as well as the best purely therapeutic alternative, and that they should not be prepared to blithely trade therapeutic benefits for enhancement ones (although doing so would seem unlikely to yield a favorable RBR). However, the standard problem with interventions that violate clinical equipoise seems to be that they jeopardise the full therapeutic benefits a subject stands to gain, or at least introduce additional risks, without providing any compensatory benefit. Yet this is not the case of therapeutic enhancements. It does not seem to us that it must be unethical to allow subjects with medical needs to freely decide to run such potential additional risks when enhancement benefits are also at stake.

\subsection{Therapeutic enhancements are less reversible than pure ones}

Some might argue, against our proposal, that military enhancements should always be reversible, either because permanent enhancements might present unacceptable risks for their recipients (such as blood leakage and other safety issues, in the case of invasive BCIs), or from a very different perspective, because they might confer an unfair advantage, for instance in the employment context, once a subject had returned to civilian life (an idea mentioned by Thorpe et al., 2017). However, the argument might go on, therapeutic enhancements are by their very nature irreversible, insofar as their removal would go against the medical needs of the recipient, and would on that account be indefensible. Therefore, one might conclude, military enhancements should be directly tested on healthy subjects, who would not unacceptably suffer from having them removed once the enhancements were no longer needed (whether for 
research or actual combat purposes).

While this line of argument is not entirely without merit, it nonetheless seems to rely on some overly strong assumptions. It is thus not true that removing a therapeutic enhancement would necessarily entail ceasing to meet the medical needs of the recipient. In some cases, the enhancement could simply be replaced by a purely therapeutic alternative: if, say, a "super" artificial organ turned out to present health risks beyond a certain period of use, it could then be swapped for a "normal", safer organ, whether artificial or natural. (Of course, this new construct would need to be safer for long-term use than the original enhancement, otherwise considerations of safety will not support preferring a normal replacement to an enhancing one.) Admittedly, this might not be possible in other cases. For instance, were a BCI to simultaneously fulfill both a therapeutic function (e.g. restoring control of devices like a computer) and an enhancing one (say, enabling silent telepathic communication) in a patient, swapping it for a new device that only fulfilled the former function might not be an option, as the prospects of replacing a brain implant with a new one are poor, at least for now, ${ }^{6}$ and it is not clear what therapeutic equivalent could be provided, say, to patients with tetraplegia (as long as a full-fledged cure for the condition remains out of reach). Even so, however, it is not clear that this must pose an insurmountable problem. Assuming such polyvalent invasive BCIs were to become reality, they could presumably be designed so as to allow for the selective disabling of their enhancing functions - if deemed appropriate - without compromising the therapeutic ones and requiring a full removal. ${ }^{7}$

Moving to the argument from fairness, we would argue that the competitive edge some veterans might enjoy from their enhancements once they had returned to civilian life could in principle be justified as an appropriate reward for their service. How plausible such a justification would be will depend on the details of the case, including the magnitude of the said advantage, and thus cannot be assessed in the abstract. This justification would admittedly not apply to research subjects who are not members of the military - although one might perhaps construct an analogous argument in their favor, citing the need to reward voluntary participation in potentially risky research. The costs of granting a person indefinite access to a therapeutic

\footnotetext{
${ }^{6}$ Dr Frederic Gilbert, personal communication.

7 True, current invasive BCIs gradually lose their effectiveness over time, which requires their removal after a few years of use. However, this is a general issue with invasive BCIs, and not one that specifically affects those having enhancement as their ultimate purpose.
} 
enhancement, which might be high, would also be a relevant issue. However, this consideration will only provide plausible grounds for discontinuing such an intervention (assuming it is paid for through public funds), and replacing it with a purely therapeutic one, if the costs imposed by the former are significantly higher than those of the latter. Whether or not this will apply in any concrete case remains to be seen, and cannot be assumed in advance.

\subsection{Incentivizing risky or deceitful behavior}

Another potential concern might be that if people, including soldiers, affected by a pathological condition were known to be regarded as research subjects of choice for enhancement research, this would risk encouraging certain forms of undesirable behavior among healthy subjects. Some might thus try and fake the symptoms of the condition that provided the gateway to the relevant trials. Besides being an ethically objectionable practice, it might also undermine the reliability of the research in question. Others might go further and, if not actively harm themselves, at least deliberately engage in unnecessarily risky behavior, whether in situations of combat or during training, with the hope of ultimately ending up enhanced. Testing enhancement interventions on healthy subjects, by contrast, would not create such perverse incentives.

Considering first the concern about incentivizing reckless risk-taking, it strikes us as rather speculative. Most people, and most members of the armed forces, are surely not likely to choose to expose themselves to avoidable risks to their life and health for the sake of merely possible, and rather elusive, enhancement benefits. And the few who might be inclined to do so do not seem to present a serious ethical concern. Soldiers who engage in needlessly risky conduct, potentially compromising the success of combat missions and the safety of their comrades, can be sanctioned in accordance with military law. (And of course, not all forms of risky behavior need be counterproductive in this way; some can be heroic and thus praiseworthy.) Furthermore, those who ended up harming themselves as a result of their reckless behavior would ultimately be responsible for it, as long as they had not been pressured into such behavior by their superiors.

The possibility that some prospective research subjects might fake the symptoms of a particular condition, in order to become eligible for a therapeutic enhancement trial, seems somewhat 
more realistic, although again it is not clear that we should expect this to become a widespread phenomenon. For some conditions, such as limb loss, such deception will clearly not be possible. In other cases, such as pathologies of mental (e.g. memory) functioning, it might be more practicable. Two main concerns would then arise: first, the impact of the deception on the validity of the research results, since it could lead to an overestimation of the enhancement potential of the relevant intervention; and secondly, the possible extra risks for the research subject resulting from being mistakenly treated as suffering from a pathology (and possibly exposed to a more aggressive intervention than would otherwise be the case). Of course, one might again argue that those risks would be self-inflicted, and therefore less of a concern. Still, these issues about personal responsibility notwithstanding, efforts should be made to avoid both of these undesirable outcomes. This might be achieved by considering all possible ways of supplementing a patient's self-reports with more objective measures of a particular condition, derived for instance from medical imaging.

\subsection{Military enhancement and risk beyond research subjects}

The final challenge we will consider to our line of argument stresses that it focuses exclusively on the risks and benefits that might accrue to research subjects in enhancement trials. However, one might argue, such considerations extend beyond that particular scope, to society as a whole. There is thus a general concern that promoting the development of "super soldiers", whether via therapeutic or pure enhancements, will lead to an enhancement arms race that is likely to make conflicts even fiercer in nature, thereby increasing the risks to civilian populations and raising the pressure for ever more effective enhancement, irrespective of RBR. The latter point in turn suggests that while promoting the best possible RBR for subjects in enhancement research is certainly a worthy goal, the greater restrictions that this approach would impose on such research might also place the nations that followed it at a disadvantage towards rivals that did not share similar qualms, and consequently did not hesitate to test cutting-edge military enhancements directly on healthy subjects.

In response, while it is certainly legitimate to worry about the potential harms that might result from an enhancement arms race for both soldiers and non-combatants, we may note that this concern is not exclusive to military enhancement research. Rather, it applies more broadly to advances in military technology, and in particular to those with great potential for destruction, 
such as nuclear or biological weapons. All of these cases raise the major challenge of how to pursue international efforts that might successfully put a stop to such an arms race, and persuade the great powers that they could forfeit further developments in the relevant areas without unacceptably endangering their national security. In any case, it seems unlikely at the present stage that an enhancement arms race would present a threat comparable to an arms race involving weapons of mass destruction, or military applications of artificial intelligence such as $\mathrm{AI}$-augmented cyber warfare or autonomous weapons. ${ }^{8}$

The possibility of an "ethics gap" (Boudreaux, 2019) or "bad guy advantage" in the context of military enhancement research is also a tricky issue. Clearly, researchers committed to upholding international standards of research ethics should not be willing to abandon them on the grounds that researchers in rival countries are willing to flout them. Here again, coordinated efforts should ideally be made to try and pressure nations around the world to adhere to such standards. Nonetheless, how rival nations behave might still unavoidably impact what it is reasonable for a particular country to do. For instance, even though avoiding invasive interventions when looking for new ways to enhance mental functioning in soldiers might be a desirable policy, it might be too risky to rigidly stick to it if it turned out that invasive enhancements have a persistent edge over non-invasive ones, and that rival militaries were embracing the former. Given this, we may certainly hope that, as some anticipate (Cinel et al., 2019), the risk profile of invasive BCIs will improve with further technological advances, or alternatively, that closing the gap in performance between invasive and non-invasive interventions into the brain will turn out to be technically feasible.

\section{Conclusions}

While not the only relevant consideration when it comes to the ethics of military enhancement research, the RBR for research subjects of the interventions being developed and tested is nonetheless of clear significance. In our discussion of this issue, we have started from a commonly held principle about the typical RBR of therapies vs. enhancements, which we have referred to as the Assumption. We have argued that, in its standard formulation, this principle

\footnotetext{
${ }^{8}$ Even though the use of BCIs for human enhancement could arguably be described as an application of AI, with considerable long-term potential.
} 
is untenable. We have then sought to reformulate the Assumption, with the goal of preserving the grain of truth it contains, while avoiding its shortcomings, and particularly its presupposition of a false dichotomy between therapy and enhancement. We have also proposed additional general guidelines for the ethics of military enhancement research, including one that singles out a certain type of therapeutic enhancements as providing a more responsible path to human trials of the relevant (often, though not always, invasive) interventions than pure enhancement applications. We have considered some potential objections to our proposal. While acknowledging their potential insights (which partly depend on the future trajectory of interventions like invasive BCIs), we have ultimately found them to be unpersuasive, at least provided that it is understood as fully non-coercive towards the candidates for such therapeutic enhancement trials.

On those grounds, we consider our proposed guidelines to provide a superior alternative to the Assumption. That being said, we agree, first, that Mehlman and Berg are right to emphasize the need to take into account "the specifics of the study in question" when assessing the RBR of any proposed intervention (Mehlman and Berg, 2008, p. 550). We have also noted that there is a strong reason for militaries to seek to perfect non-invasive enhancements in preference to invasive ones, insofar as it is possible to do so without impeding the ultimate performance of soldiers. Going beyond this idea, one might further argue that the army's ultimate goal should be to take humans away from the battlefield completely, and replace them with remotecontrolled weapons, such as drones and military robots. While such a development certainly sounds desirable from the perspective of minimizing risks to troops, it also raises significant ethical issues, such as potentially greater threats to civilians, as well as issues of accountability if autonomous killing machines start replacing human soldiers. As important as these issues might be, they lie beyond the scope of the present paper.

\section{References:}

AGAR, N. 2004. Liberal Eugenics : in Defence of Human Enhancement, Malden, Mass. ; Oxford, Blackwell Publishing.

BOUDREAUX, B. 11/01/2019 2019. Does the U.S. Face an AI Ethics Gap? The RAND Blog [Online]. Available from: https://www.rand.org/blog/2019/01/does-the-us-face-an-aiethics-gap.html 2019]. 
BROCK, D. 1998. Enhancements of Human Function: Some Distinctions for Policymakers. In: PARENS, E. (ed.) Enhancing Human Traits: Ethical and Social Implications. Washington, DC: Georgetown University Press, 48-69.

CAMPOBASSO, T. 2015. Super Soldiers: 3D Bioprinting and the Future Fighter. Small Wars Journal [Online]. Available: http://smallwarsjournal.com/jrnl/art/super-soldiers3d-bioprinting-and-the-future-fighter [Accessed 05/12/2017].

CINEL, C., VALERIANI, D. \& POLI, R. 2019. Neurotechnologies for Human Cognitive Augmentation: Current State of the Art and Future Prospects. Front Hum Neurosci, $13,13$.

DARPA. 2018. Progress in Quest to Develop a Human Memory Prosthesis [Online]. Available: https://www.darpa.mil/news-events/2018-03-28 [Accessed 04/08/2019 2019].

DARPA. 2019. Six Paths to the Nonsurgical Future of Brain-Machine Interfaces [Online]. Available: https://www.darpa.mil/news-events/2019-05-20 [Accessed 20/08/2019 2019].

ERLER, A. 2017. The Limits of the Treatment-Enhancement as a Guide to Public Policy. Bioethics, 31, 608-615.

GREENE, M. \& MASTER, Z. 2018. Ethical Issues of Using CRISPR Technologies for Research on Military Enhancement. Journal of Bioethical Inquiry, 15, 327-335.

LIN, P., MEHLMAN, M. \& ABNEY, K. 2013. Enhanced Warfighters: Risk, Ethics, and Policy. The Greenwall Foundation.

MCGEE, E. M. \& MAGUIRE, G. Q., JR. 2007. Becoming Borg to Become Immortal: Regulating Brain Implant Technologies. Camb Q Healthc Ethics, 16, 291-302.

MEHLMAN, M. J. 2015. Captain America and Iron Man: Biological, Genetic and Psychological Enhancement and the Warrior Ethos. In: LUCAS, G. (ed.) Routledge Handbook of Military Ethics. London; New York: Routledge, 406-20.

MEHLMAN, M. J. \& BERG, J. W. 2008. Human Subjects Protections in Biomedical Enhancement Research: Assessing Risk and Benefit and Obtaining Informed Consent. Journal of Law, Medicine and Ethics, 36, 546-49.

MEHLMAN, M. J. \& LI, T. Y. 2014. Ethical, Legal, Social, and Policy Issues in the Use of Genomic Technology by the U.S.Military. J Law Biosci, 1, 244-80.

NUYUJUKIAN, P., ALBITES SANABRIA, J., SAAB, J., PANDARINATH, C., JAROSIEWICZ, B., BLABE, C. H., FRANCO, B., MERNOFF, S. T., ESKANDAR, E. N., SIMERAL, J. D., HOCHBERG, L. R., SHENOY, K. V. \& HENDERSON, J. M. 2018. Cortical Control of a Tablet Computer by People with Paralysis. PLoS One, 13, e0204566.

OFFICE OF THE UNDER SECRETARY OF DEFENSE FOR PERSONNEL AND

READINESS. 2018. DoD Instruction 6130.03: Medical Standards for Appointment, Enlistment, or Induction into the Military Services. Available: https://www.med.navy.mil/sites/nmotc/nami/arwg/Documents/WaiverGuide/DODI_6 130.03_JUL12.pdf [Accessed 23/08/2019].

PELTIER, C. \& PETTIJOHN, K. 2018. The Future of Steroids for Performance Enhancement in the U.S. Military. Mil Med, 183, 151-153.

SAVULESCU, J., SANDBERG, A. \& KAHANE, G. 2011. Well-Being and Enhancement. In: TER MEULEN, R., SAVULESCU, J. \& KAHANE, G. (eds.) Enhancing Human Capacities. Oxford: Wiley-Blackwell, 3-18.

THORPE, J. B., GIRLING, K. D. \& AUGER, A. 2017. Maintaining Military Dominance in the Future Operating Environment: A Case for Emerging Human Enhancement Technologies that Contribute to Soldier Resilience. Small Wars Journal [Online]. 
Available: https://smallwarsjournal.com/jrnl/art/maintaining-military-dominance-inthe-future-operating-environment-a-case-for-emerging-huma [Accessed 29/08/2019]. WEIJER, C. \& MILLER, P. B. 2004. When Are Research Risks Reasonable in Relation to Anticipated Benefits? Nature Medicine, 10, 570-573.

WOLBRING, G., DIEP, L., YUMAKULOV, S., BALL, N., LEOPATRA, V. \& YERGENS, D. 2013. Emerging Therapeutic Enhancement Enabling Health Technologies and Their Discourses: What Is Discussed within the Health Domain? Healthcare (Basel), $1,20-52$. 